engine of each creation. We apply the rule that, to turn science into emotion, the philosophical implications of scientific ideas must always make up the backbone of each plot.

For example, the topic of quantum mechanics becomes intimately linked with the desire to quench one's personal failures and grief in a 'probability space'; the concept of 'time' links with the very human question, "Where does passing time go?"; Voyager 1's iconic Pale Blue Dot picture offers the opportunity to delve into the mechanisms of climate change and how our pale blue dot will look in a not-so-far future. Each production explores ways to turn one or more scientific topics into poignant existential questions.

Our work lies at the interface between science and art, human beings and society, and the philosophy of science. The project involves professional performing artists, researchers, science educators and facilitators to promote science engagement with students and general audiences.

The plays that we have created have been performed hundreds of times around the world. Their topics so far encompass quantum mechanics, thermodynamics, brain physiology, artificial intelligence, climate change, Moon and space exploration, and time. Collaborations with several universities and artistic institutions are under way, as the project aims to become a useful tool for outreach, science communication and science education research. We investigate ways to turn scientific storytelling into a structured and engaging art.

\section{Andrea Brunello ${ }^{1,2 \star}$, Pierre Echard and Stefano Oss ${ }^{2}$ \\ ${ }^{1}$ Arditodesio Theatre Company, Trento, Italy. ${ }^{2}$ Department of Physics, University of Trento, Trento, Italy. \\ *e-mail: a.brunello@jetpropulsiontheatre.org}

Published online: 7 May 2019

https://doi.org/10.1038/s41565-019-0445-7

\title{
Perceptions at the nanoscale
}

\author{
Artistic imagination can help drive scientific imagination.
}

remember working with an artist

(Becca Rose) to produce a cover

illustration for one of my papers. She made a watercolour image entitled Molecular Energy Flow. As an artist, Becca's decisions were naturally unrestrained by conventional scientific aesthetics. And this came as a small shock to some of my colleagues, who observed that her image did not represent 'what molecules look like'. Apart from the ontological inconsistency of this statement (nobody can see molecules!), their reaction highlights the misalignment between the molecular aesthetic in Becca's image and the aesthetic that scientists carry around in their heads when they imagine molecules.

My colleagues' reactions to works of art inspired by scientific concepts are not uncommon and neatly illustrate the extent to which aesthetic projections construct scientific imagination. Consciously and unconsciously, scientists cling to aesthetic projections, because they enable them to cognize domains that are otherwise imperceptible. This is especially true at the nanoscale, because it is a domain that is inaccessible to our sensory organs; here, progress depends on our ability to construct aesthetic projections that our brains can effectively parse and process. In many cases, the act of aesthetic projection is as important as the experimental data themselves: in shaping our scientific imagination, it focuses our data-gathering efforts.

Humans are sophisticated sensory machines capable of simultaneously integrating complex data across multiple perceptual channels, including the visual,

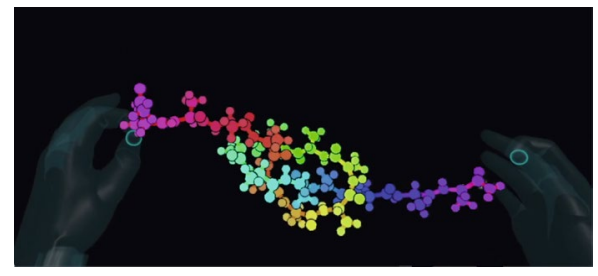

A virtual reality glove that enables scientists to use their proprioceptive sense to 'reach out and feel' molecules.

auditory, olfactory and somatosensory cortices. Nevertheless, for the nanoscale, the representations we make are primarily designed for parsing by our visual cortex (two-dimensional plots, images and movies), drawing on an aesthetic that tends to be arbitrary and limited - for example, 'balland-stick' representations of molecules.

Building on psychology and neuroscience research linking multisensory processing and attention, our laboratory actively integrates both artistic and scientific practices to develop technologies for enabling multisensory perception of nanoscale dynamics, exploring perceptual channels beyond vision - for example, audio, touch and proprioception. For instance, our flexible, open-source, multi-person virtual reality software framework 'Narupa' (https:// irl.itch.io/narupaxr) enables groups of people to simultaneously cohabit real-time simulation environments where they can 'reach out and feel' the rigorous dynamics of nanoscale structures, interactively visualizing, hearing and manipulating them with atomic-level precision. Some of the biggest challenges we face building this framework involve designing aesthetic projections that enable multimodal forms of sensory perception for nanoscale objects that we cannot directly experience. In this respect, close collaboration between artists and scientists is crucial, because it encourages a kind of positive feedback loop where both kinds of enquiry drive one another to explore new domains: the science inspires artistic imagination, and the artistic imagination then drives technological developments that enable fresh scientific approaches, which then again drives the artistic imagination.

Our work has highlighted a number of applications where multisensory approaches enable more intuitive (and efficient!) nanoscale design and engineering. This is a relatively unexplored territory for nanotechnology research, requiring deep and symmetric exchanges between aesthetic practice and scientific practice, but it has the potential to usher in a new paradigm for nano-engineering, improving our dynamical intuition and our ability to communicate.

For more information, see https://glowwacky.com.

\section{David R. Glowacki}

Scientist, artist and cultural theorist, University of Bristol, Bristol, UK.

e-mail:glowacki@bristol.ac.uk

Published online: 7 May 2019

https://doi.org/10.1038/s41565-019-0452-8 